\title{
Ru-catalyzed reverse water gas shift reaction with near-unity selectivity and superior stability
}

Rui Tang, ${ }^{1, \dagger}$ Zhijie Zhu, ${ }^{1, \dagger}$ Chaoran Li, ${ }^{1, *}$ Mengqi Xiao, ${ }^{1}$ Zhiyi Wu, ${ }^{1}$ Dake Zhang, ${ }^{1}$

Chengcheng Zhang, ${ }^{1}$ Yi Xiao, ${ }^{1}$ Mingyu Chu, ${ }^{1}$ Alexander Genest, ${ }^{2}$ Günther Rupprechter, ${ }^{2}$

Liang Zhang, ${ }^{1}$ Xiaohong Zhang, ${ }^{1}$ * Le He ${ }^{1, *}$

${ }^{1}$ Institute of Functional Nano \& Soft Materials (FUNSOM), Jiangsu Key Laboratory for Carbon-Based Functional Materials \& Devices, Joint International Research Laboratory of Carbon-Based Functional Materials and Devices, Soochow University, Suzhou, Jiangsu 215123, China

${ }^{2}$ Institute of Materials Chemistry, Technische Universität Wien, Vienna 1060, Austria

†These authors contributed equally to this work

*Email: crli@suda.edu.cn; xiaohong_zhang@suda.edu.cn; lehe@suda.edu.cn 


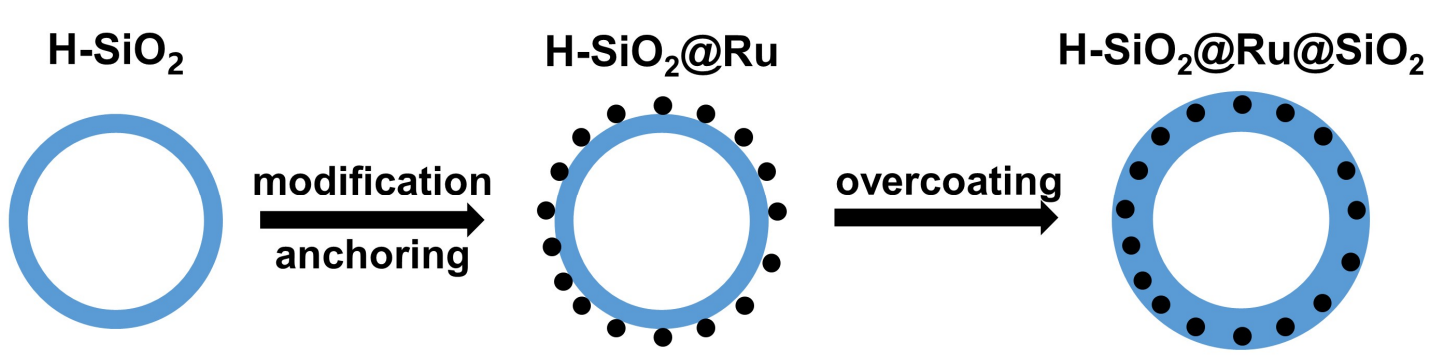

Figure S1. Schematic illustration of the synthesis process of $\mathrm{H}-\mathrm{SiO}_{2} @ \mathrm{Ru} @ \mathrm{SiO}_{2}$ nanostructures. 


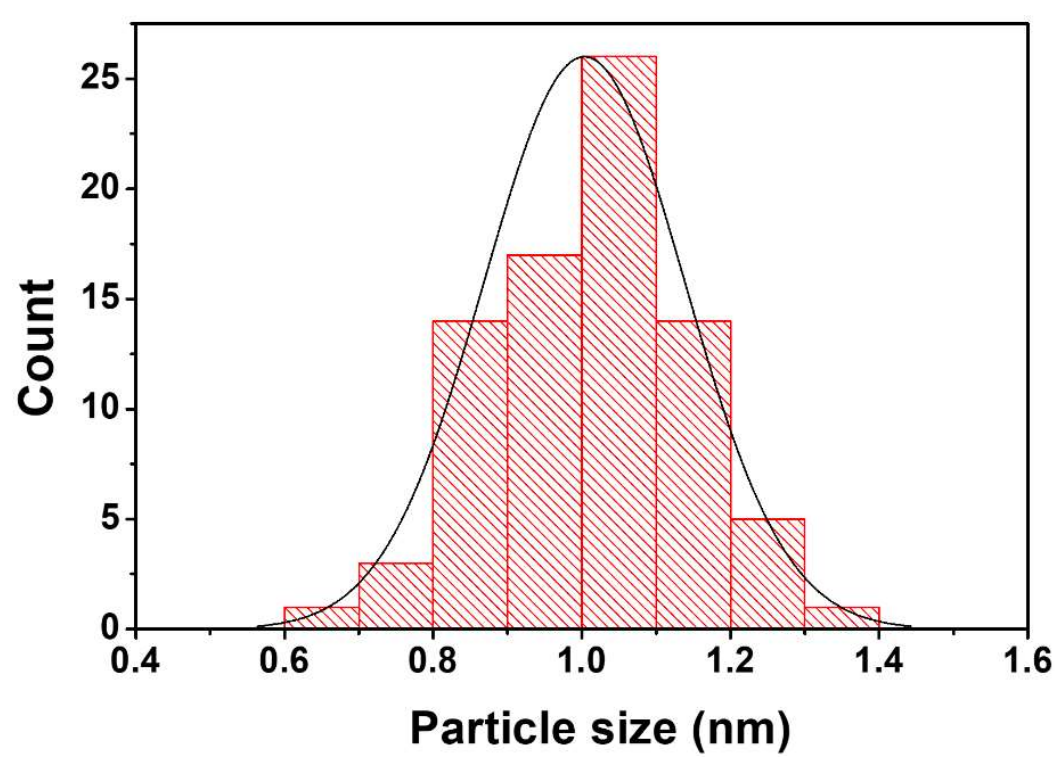

Figure S2. Size distribution of freshly prepared $\mathrm{H}-\mathrm{SiO}_{2} @ \mathrm{Ru}$. 
Supporting Information

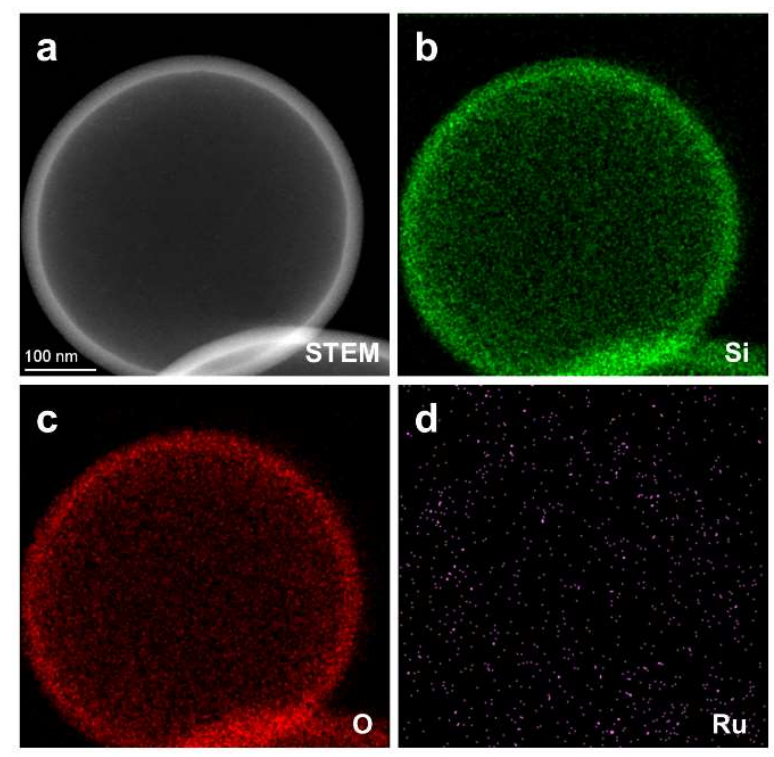

Figure S3. EDS elemental mapping images of $\mathrm{H}_{-} \mathrm{SiO}_{2} @ \mathrm{Ru}$. 


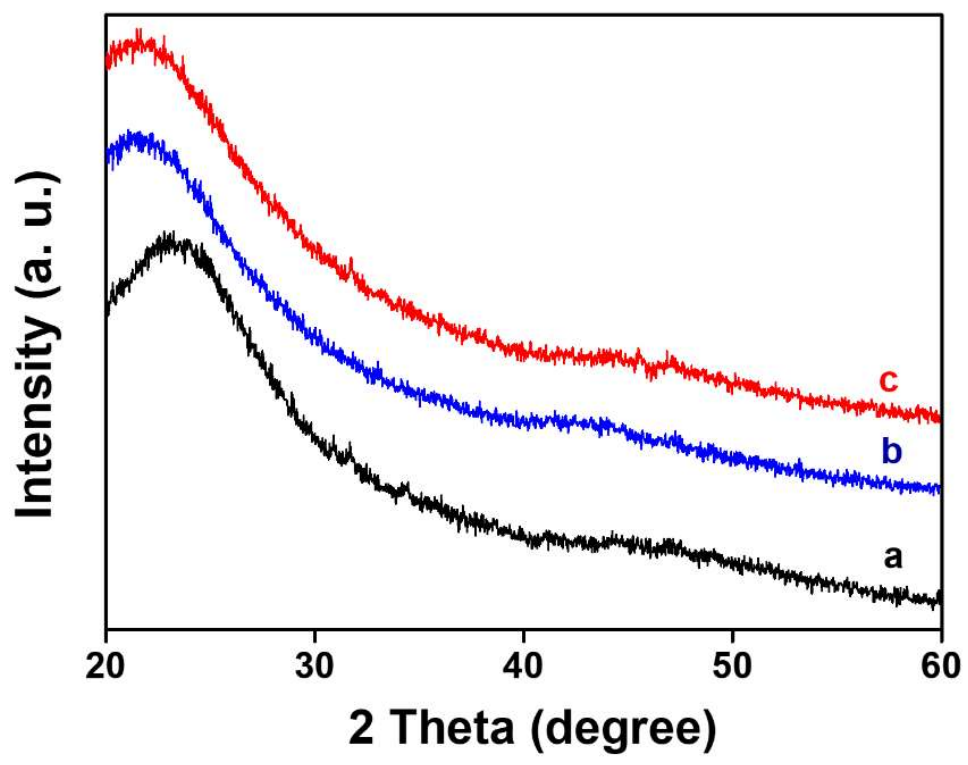

Figure S4. XRD patterns of $\mathrm{H}-\mathrm{SiO}_{2} @ \mathrm{Ru}$ catalysts: (a) freshly prepared catalyst, (b) reduced catalyst and (c) spent catalyst. 




Figure S5. Size distribution of freshly prepared $\mathrm{H}-\mathrm{SiO}_{2} @ \mathrm{Ru} @ \mathrm{SiO}_{2}-30$. 
Supporting Information

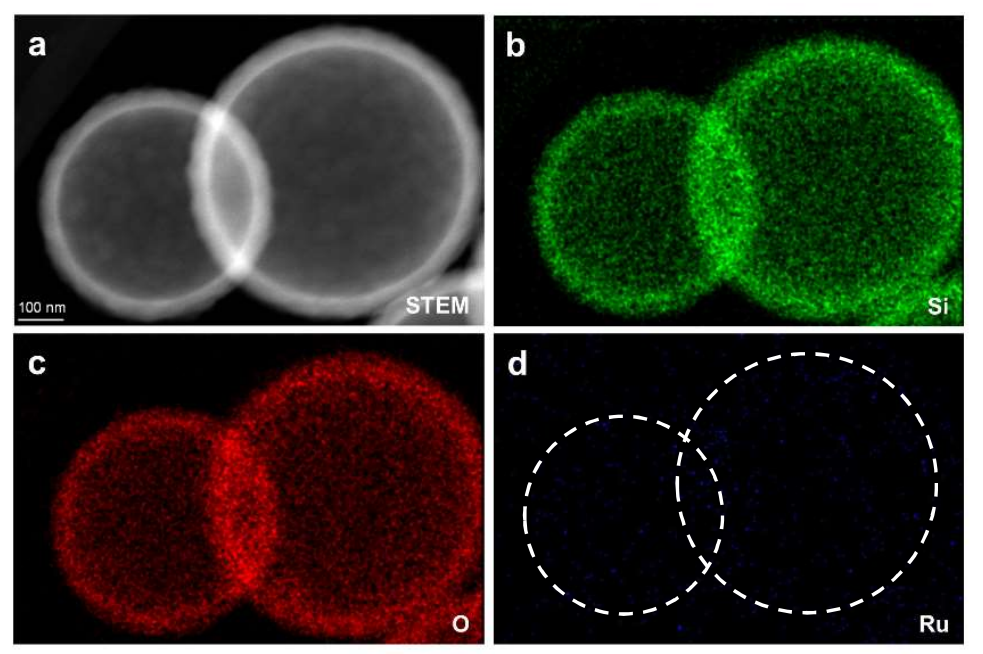

Figure S6. EDS elemental mapping images of $\mathrm{H}-\mathrm{SiO}_{2} @ \mathrm{Ru} @ \mathrm{SiO}_{2}-30$. 


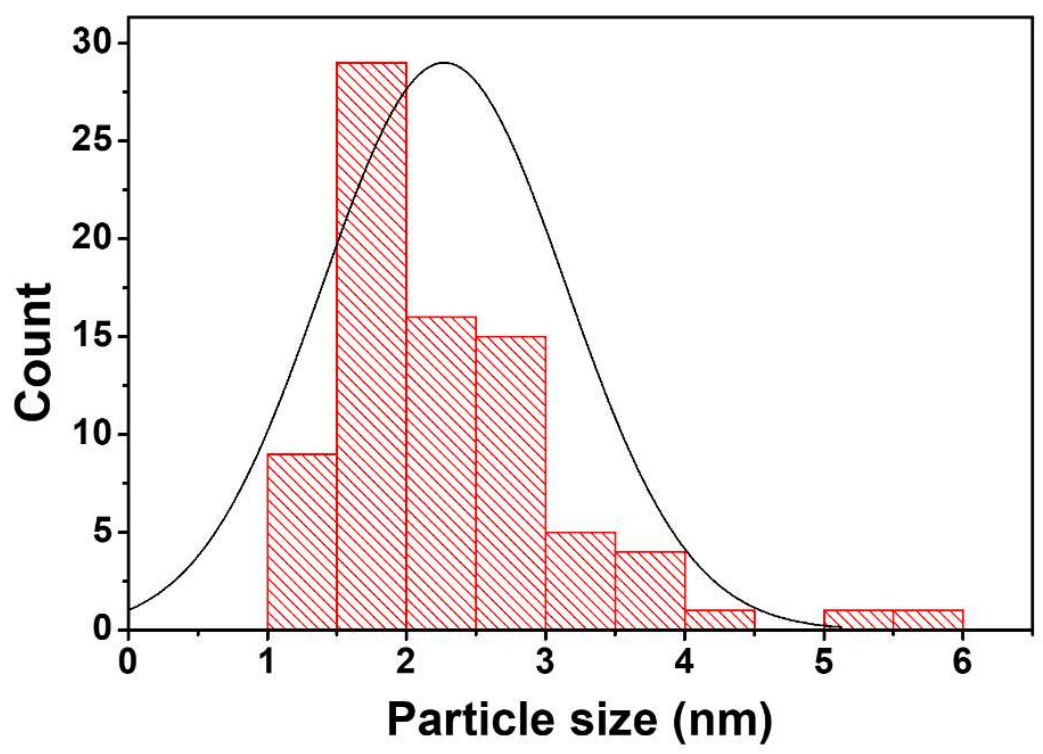

Figure S7. Size distribution of reduced $\mathrm{H}-\mathrm{SiO}_{2} @ \mathrm{Ru}-\mathrm{H}_{2}$. 


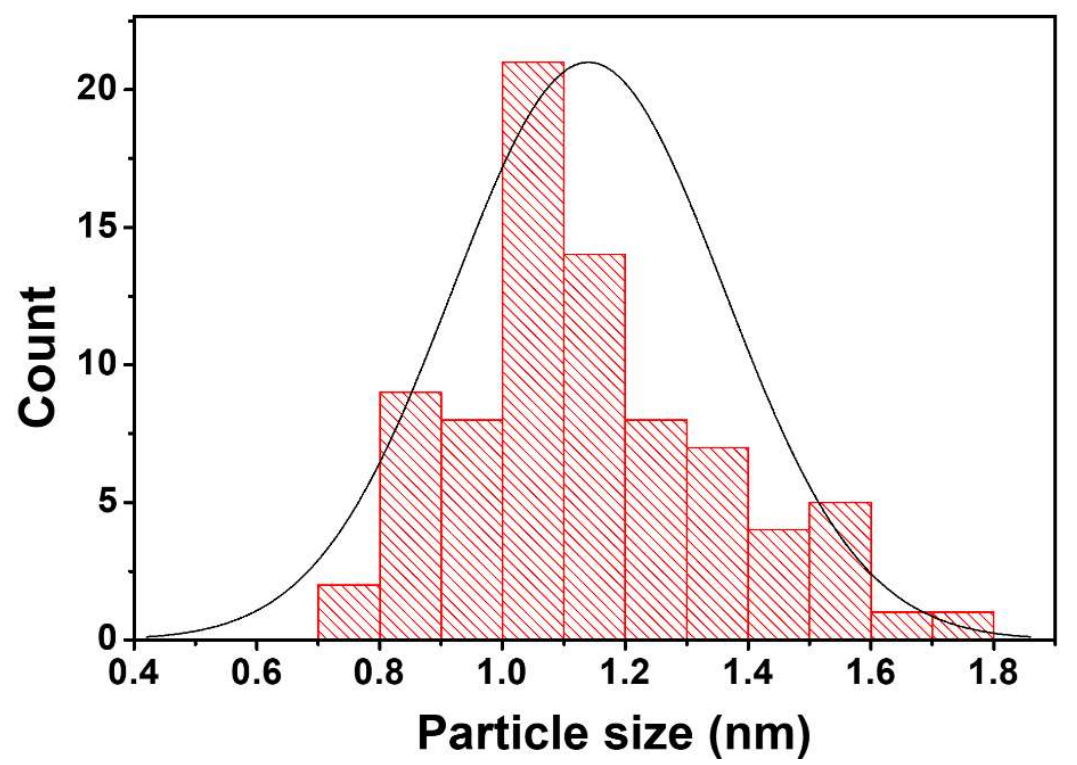

Figure S8. Size distribution of reduced $\mathrm{H}_{-} \mathrm{SiO}_{2} @ \mathrm{Ru} @ \mathrm{SiO}_{2}-30-\mathrm{H}_{2}$. 
Supporting Information



Figure S9. Thermodynamic $\mathrm{CO}_{2}$ conversion degree at atmosphere pressure. 
Supporting Information

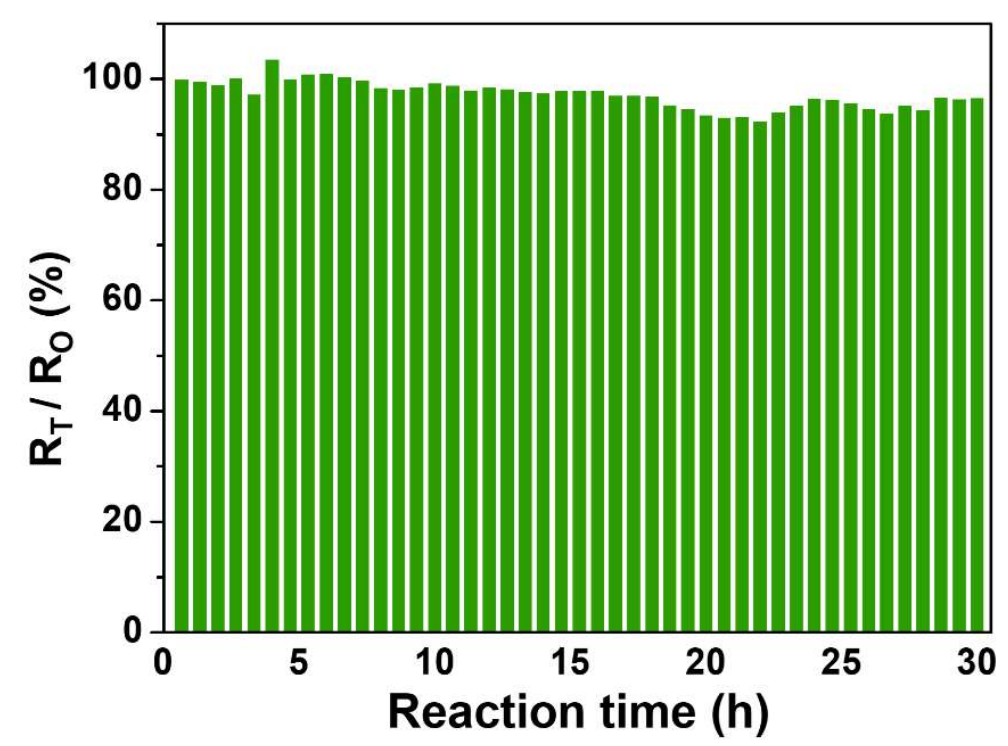

Figure S10. Catalytic activity of $\mathrm{H}_{-} \mathrm{SiO}_{2} @ \mathrm{Ru} @ \mathrm{SiO}_{2}-30-\mathrm{H}_{2}$ in a continuous 30-hour run at $400{ }^{\circ} \mathrm{C}$. 
Supporting Information

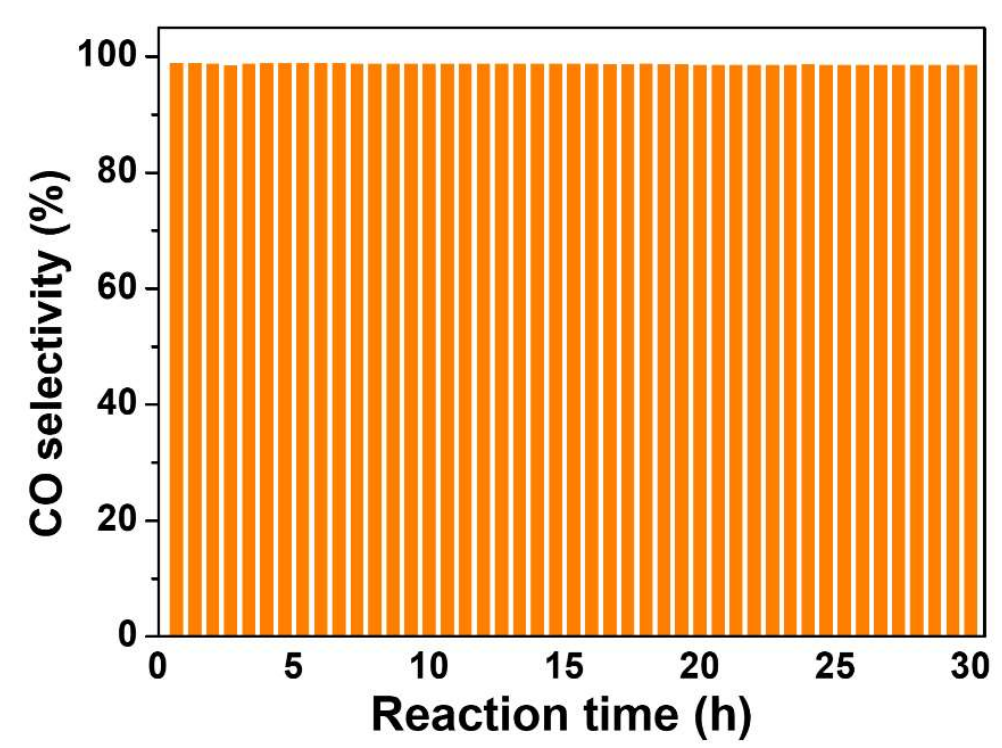

Figure S11. Catalytic selectivity of $\mathrm{H}_{-} \mathrm{SiO}_{2} @ \mathrm{Ru} @ \mathrm{SiO}_{2}-30-\mathrm{H}_{2}$ in a continuous 30hour run at $400{ }^{\circ} \mathrm{C}$. 




Figure S12. TEM image of $\mathrm{H}-\mathrm{SiO}_{2} @ \mathrm{Ru} @ \mathrm{SiO}_{2}-30-\mathrm{H}_{2}$ after testing at $400{ }^{\circ} \mathrm{C}$ for 30 hours. 
Supporting Information



Figure S13. High-resolution TEM images of $\mathrm{H}-\mathrm{SiO}{ }_{2} @ \mathrm{Ru} @ \mathrm{SiO}_{2}-30-\mathrm{H}_{2}$ after testing at $400{ }^{\circ} \mathrm{C}$ for 30 hours. 




Figure S14. Size distribution of $\mathrm{H}-\mathrm{SiO}_{2} @ \mathrm{Ru} @ \mathrm{SiO}_{2}-30-\mathrm{H}_{2}$ after testing at $400{ }^{\circ} \mathrm{C}$ for 30 hours. 


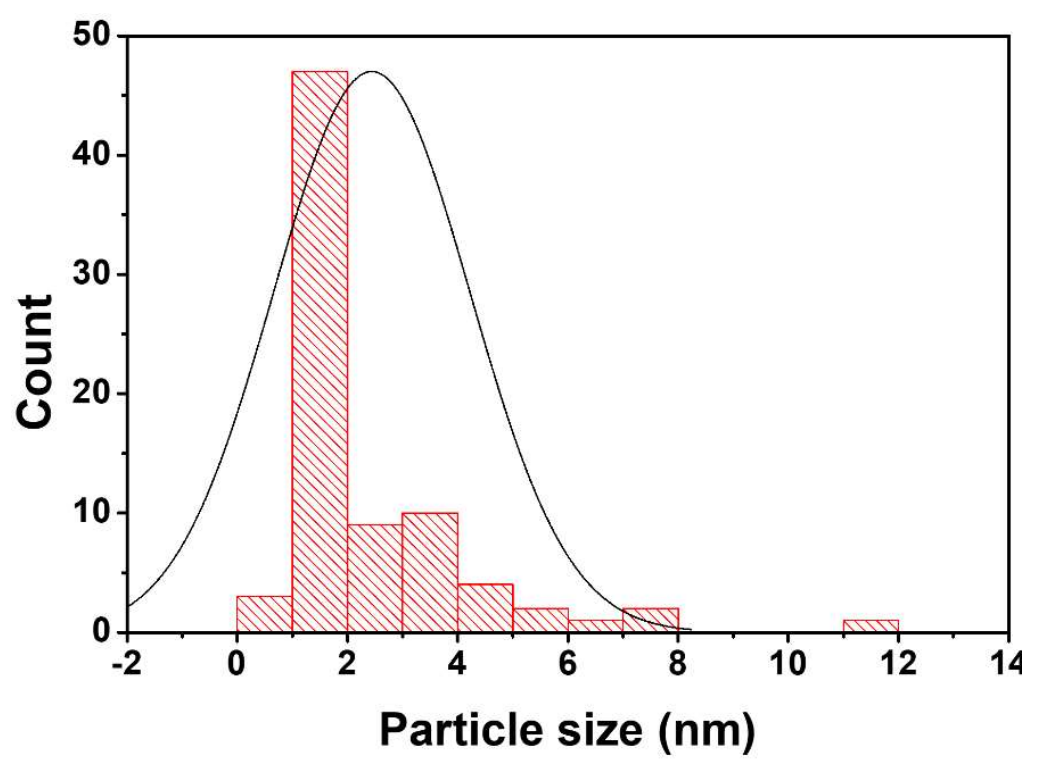

Figure S15. Size distribution of spent $\mathrm{H}-\mathrm{SiO}_{2} @ \mathrm{Ru}-\mathrm{H}_{2}$. 


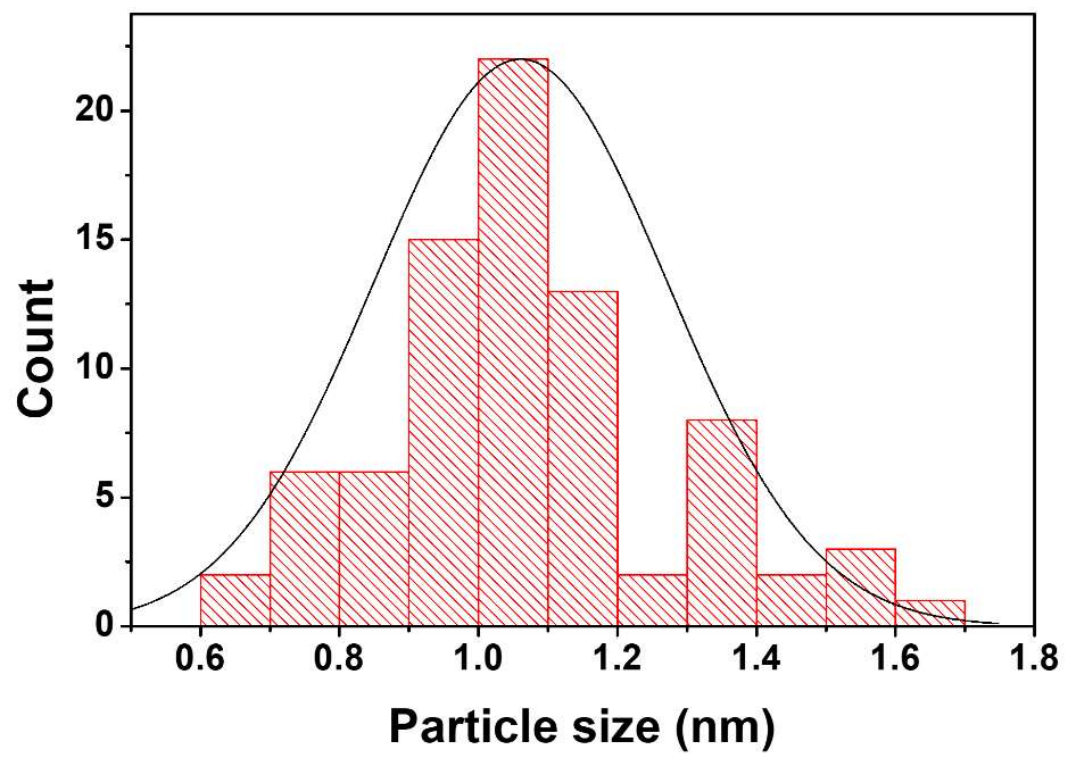

Figure S16. Size distribution of spent $\mathrm{H}-\mathrm{SiO}_{2} @ \mathrm{Ru} @ \mathrm{SiO}_{2}-30-\mathrm{H}_{2}$. 


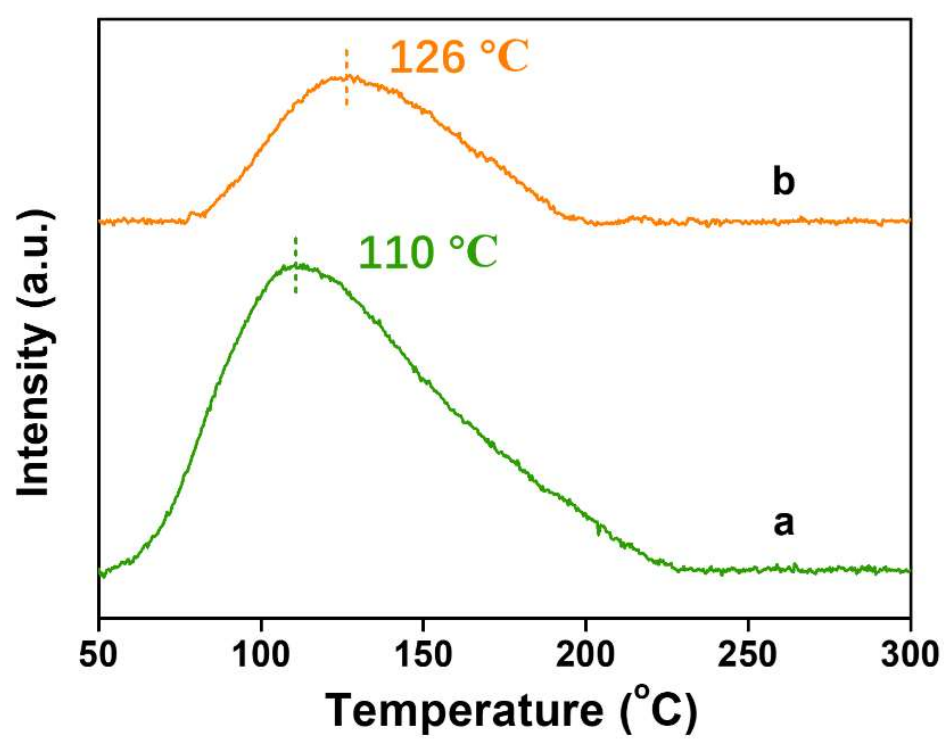

Figure S17. $\mathrm{H}_{2}-\mathrm{TPD}$ profiles of (a) $\mathrm{H}-\mathrm{SiO}_{2} @ \mathrm{Ru}-\mathrm{H}_{2}$, and (b) spent $\mathrm{H}-\mathrm{SiO}_{2} @ \mathrm{Ru}-\mathrm{H}_{2}$. 




Figure S18. Hydrogen temperature programmed desorption $\left(\mathrm{H}_{2}-\mathrm{TPD}\right)$ profile: (a) $\mathrm{H}$ $\mathrm{SiO}_{2} @ \mathrm{Ru} @ \mathrm{SiO}_{2}-30-\mathrm{H}_{2}$, (b) spent $\mathrm{H}-\mathrm{SiO}_{2} @ \mathrm{Ru} @ \mathrm{SiO}_{2}-30-\mathrm{H}_{2}$. 




Figure S19. TEM images of freshly prepared $\mathrm{H}-\mathrm{SiO}_{2} @ \mathrm{Ru} @ \mathrm{SiO}_{2}-50$. 
Supporting Information

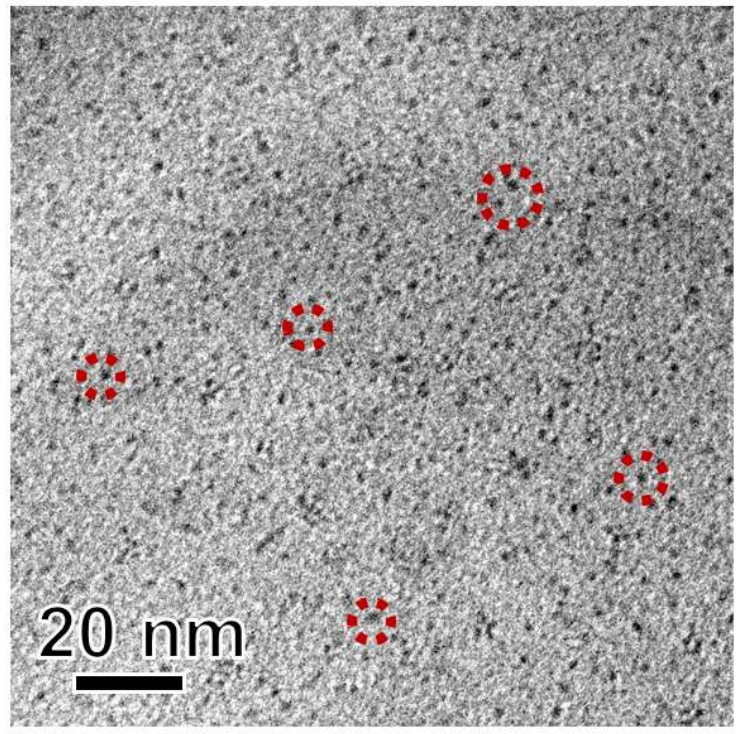

Figure S20. High-resolution TEM image of freshly prepared $\mathrm{H}-\mathrm{SiO}_{2} @ \mathrm{Ru} @ \mathrm{SiO}_{2}-50$. 


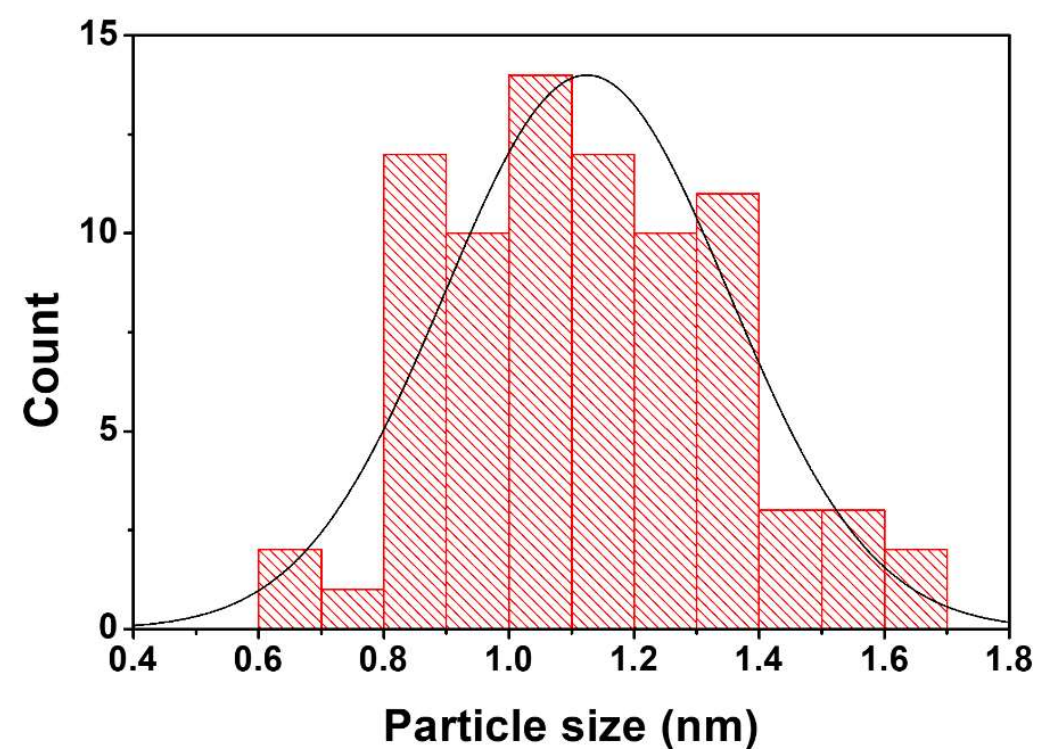

Figure S21. Size distribution of freshly prepared $\mathrm{H}_{-} \mathrm{SiO}_{2} @ \mathrm{Ru} @ \mathrm{SiO}_{2}-50$. 




Figure S22. TEM image of reduced $\mathrm{H}_{-} \mathrm{SiO}_{2} @ \mathrm{Ru} @ \mathrm{SiO}_{2}-50$, denoted as $\mathrm{H}-$ $\mathrm{SiO}_{2} @ \mathrm{Ru} @ \mathrm{SiO}_{2}-50-\mathrm{H}_{2}$. 
Supporting Information

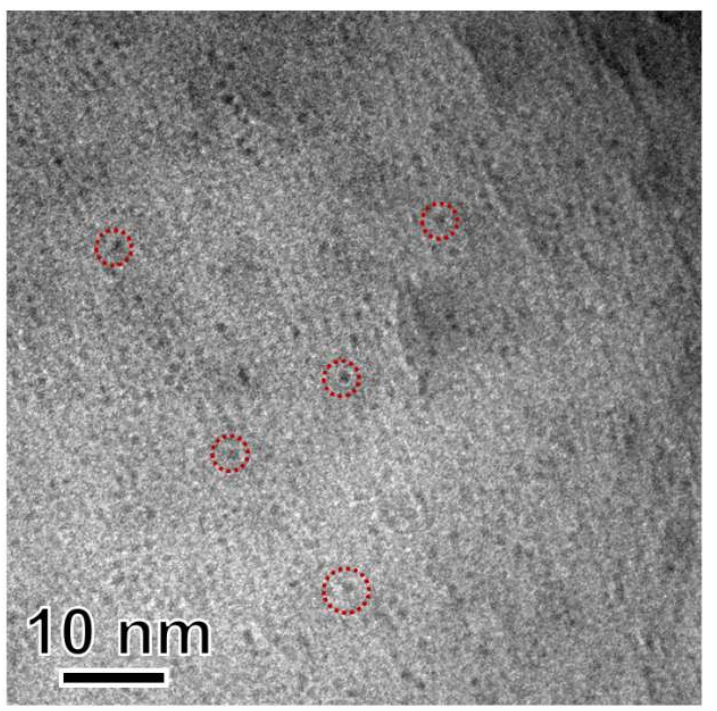

Figure S23. High-resolution TEM image of $\mathrm{H}-\mathrm{SiO}_{2} @ \mathrm{Ru} @ \mathrm{SiO}_{2}-50-\mathrm{H}_{2}$. 


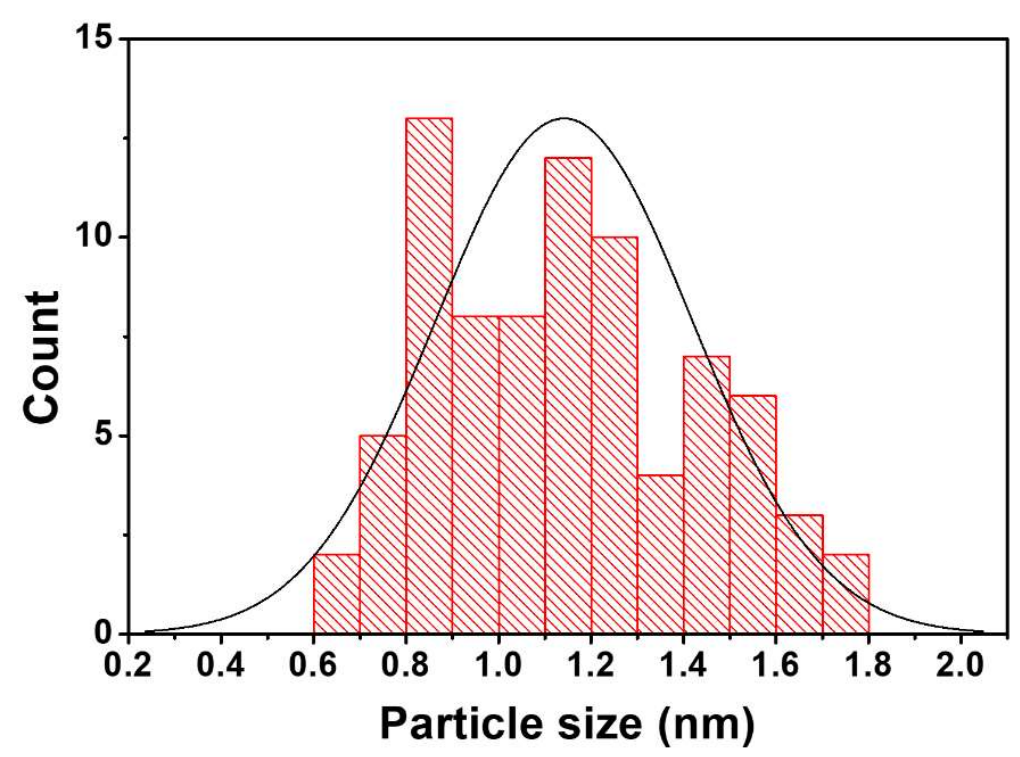

Figure S24. Size distribution of $\mathrm{H}-\mathrm{SiO}_{2} @ \mathrm{Ru} @ \mathrm{SiO}_{2}-50-\mathrm{H}_{2}$. 


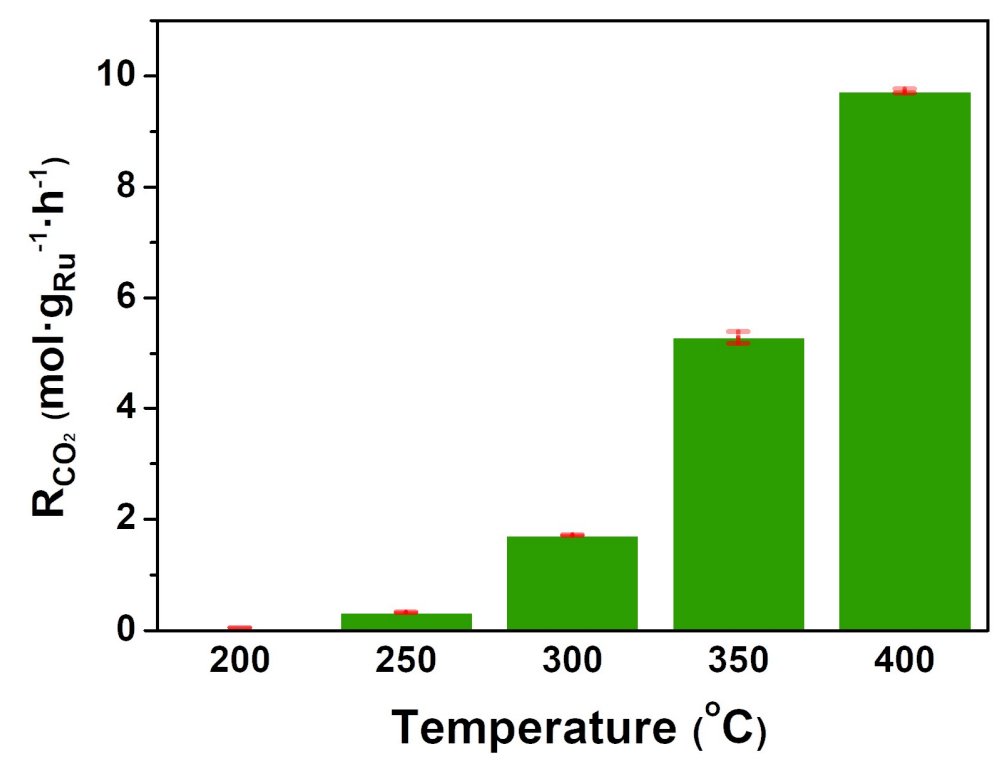

Figure S25. Temperature dependent activity of $\mathrm{H}-\mathrm{SiO}_{2} @ \mathrm{Ru} @ \mathrm{SiO}_{2}-50-\mathrm{H}_{2}$ in catalyzing $\mathrm{CO}_{2}$ hydrogenation. 




Figure S26. Temperature dependent selectivity of $\mathrm{H}_{-}-\mathrm{SiO}_{2} @ \mathrm{Ru} @ \mathrm{SiO}_{2}-50-\mathrm{H}_{2}$ in catalyzing $\mathrm{CO}_{2}$ hydrogenation. 




Figure S27. Catalytic stability of $\mathrm{H}-\mathrm{SiO}_{2} @ \mathrm{Ru} @ \mathrm{SiO}_{2}-50-\mathrm{H}_{2}$ in a continuous 12-hour run at $400{ }^{\circ} \mathrm{C}$. 




Figure S28. Catalytic selectivity of $\mathrm{H}_{-} \mathrm{SiO}_{2} @ \mathrm{Ru} @ \mathrm{SiO}_{2}-50-\mathrm{H}_{2}$ in a continuous 12-hour run at $400{ }^{\circ} \mathrm{C}$. 




Figure S29. TEM images of spent $\mathrm{H}_{-}-\mathrm{SiO}_{2} @ \mathrm{Ru} @ \mathrm{SiO}_{2}-50-\mathrm{H}_{2}$ after test at $400{ }^{\circ} \mathrm{C}$ for $12 \mathrm{~h}$. 




Figure S30. High-resolution TEM images of spent $\mathrm{H}-\mathrm{SiO}{ }_{2} @ \mathrm{Ru} @ \mathrm{SiO}_{2}-50-\mathrm{H}_{2}$ after test at $400{ }^{\circ} \mathrm{C}$ for $12 \mathrm{~h}$. 


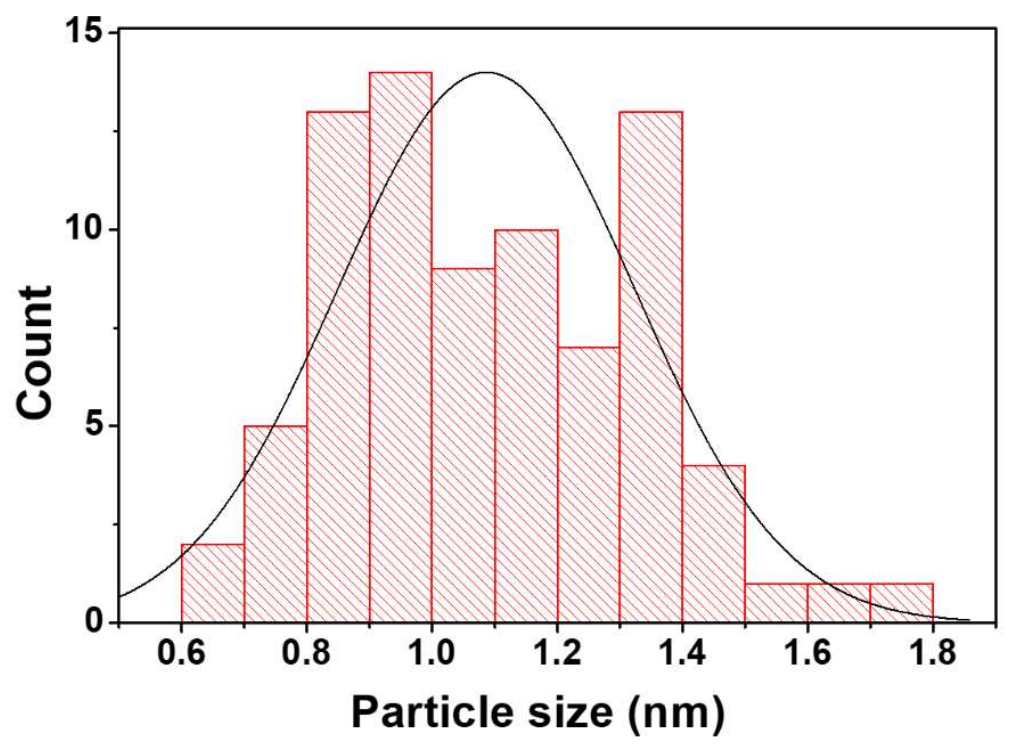

Figure S31. Size distribution of spent $\mathrm{H}-\mathrm{SiO}_{2} @ \mathrm{Ru} @ \mathrm{SiO}_{2}-50-\mathrm{H}_{2}$. 


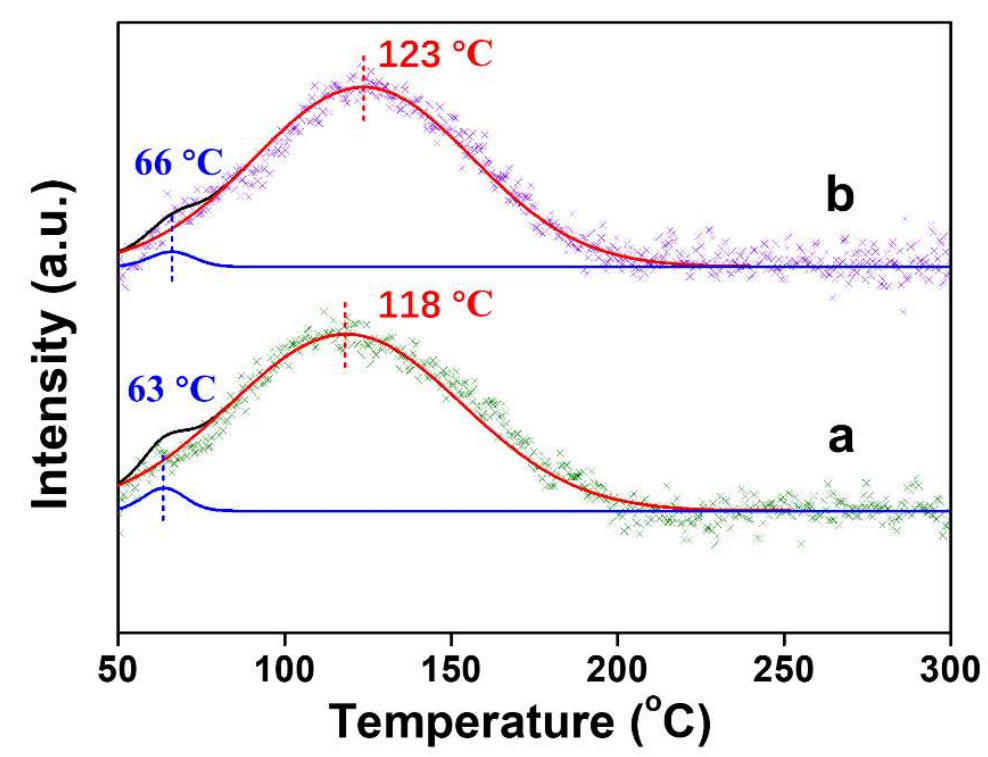

Figure S32. Hydrogen temperature programmed desorption $\left(\mathrm{H}_{2}-\mathrm{TPD}\right)$ profile: (a) H$\mathrm{SiO}_{2} @ \mathrm{Ru} @ \mathrm{SiO}_{2}-50-\mathrm{H}_{2}$, (b) spent $\mathrm{H}_{-} \mathrm{SiO}_{2} @ \mathrm{Ru} @ \mathrm{SiO}_{2}-50-\mathrm{H}_{2}$. 


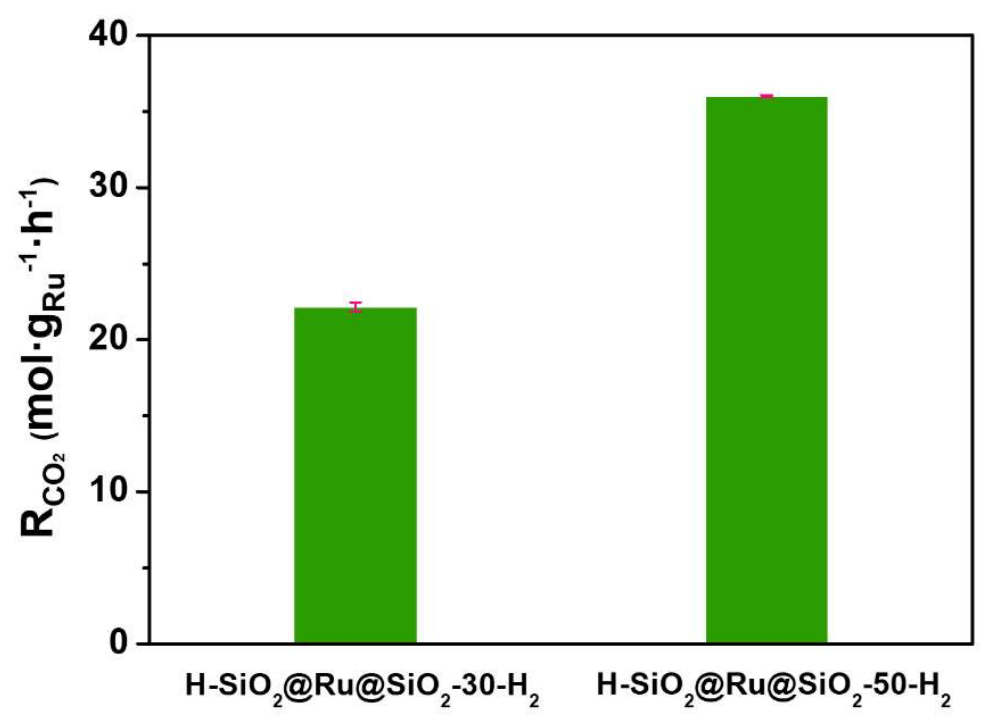

Figure S33. Catalytic activity comparison between $\mathrm{H}-\mathrm{SiO}_{2} @ \mathrm{Ru} @ \mathrm{SiO}_{2}-30-\mathrm{H}_{2}$ and $\mathrm{H}-$ $\mathrm{SiO}_{2} @ \mathrm{Ru} @ \mathrm{SiO}_{2}-50-\mathrm{H}_{2}$ at $500{ }^{\circ} \mathrm{C}$. 


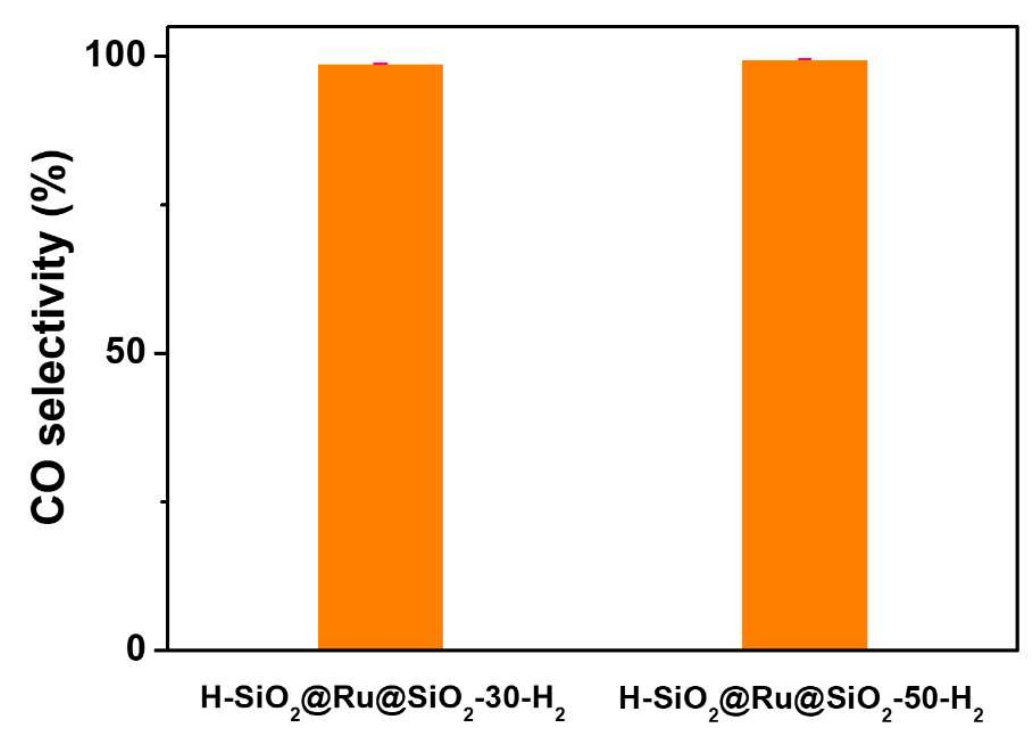

Figure S34. Catalytic selectivity comparison between $\mathrm{H}-\mathrm{SiO}{ }_{2} @ \mathrm{Ru} @ \mathrm{SiO}_{2}-30-\mathrm{H}_{2}$ and $\mathrm{H}-\mathrm{SiO}_{2} @ \mathrm{Ru} @ \mathrm{SiO}_{2}-50-\mathrm{H}_{2}$ at $500{ }^{\circ} \mathrm{C}$. 
Supporting Information



Figure S35. TEM images of $\mathrm{H}_{-}-\mathrm{SiO}_{2} @ \mathrm{Ru} @ \mathrm{SiO}_{2}-50-\mathrm{H}_{2}$ after pretreated at $800{ }^{\circ} \mathrm{C}$ in $\mathrm{H}_{2}$, denoted as $\mathrm{H}-\mathrm{SiO}_{2} @ \mathrm{Ru} @ \mathrm{SiO}_{2}-50-800$. 
Supporting Information



Figure S36. High-resolution TEM images of $\mathrm{H}-\mathrm{SiO}_{2} @ \mathrm{Ru} @ \mathrm{SiO}_{2}-50-800$. 




Figure S37. Size distribution of $\mathrm{H}_{-} \mathrm{SiO}_{2} @ \mathrm{Ru} @ \mathrm{SiO}_{2}-50-800$. 




Figure S38. Catalytic activity comparison between $\mathrm{H}-\mathrm{SiO}_{2} @ \mathrm{Ru} @ \mathrm{SiO}_{2}-50-\mathrm{H}_{2}$ and $\mathrm{H}-$ $\mathrm{SiO}_{2} @ \mathrm{Ru} @ \mathrm{SiO}_{2}-50-800$. 


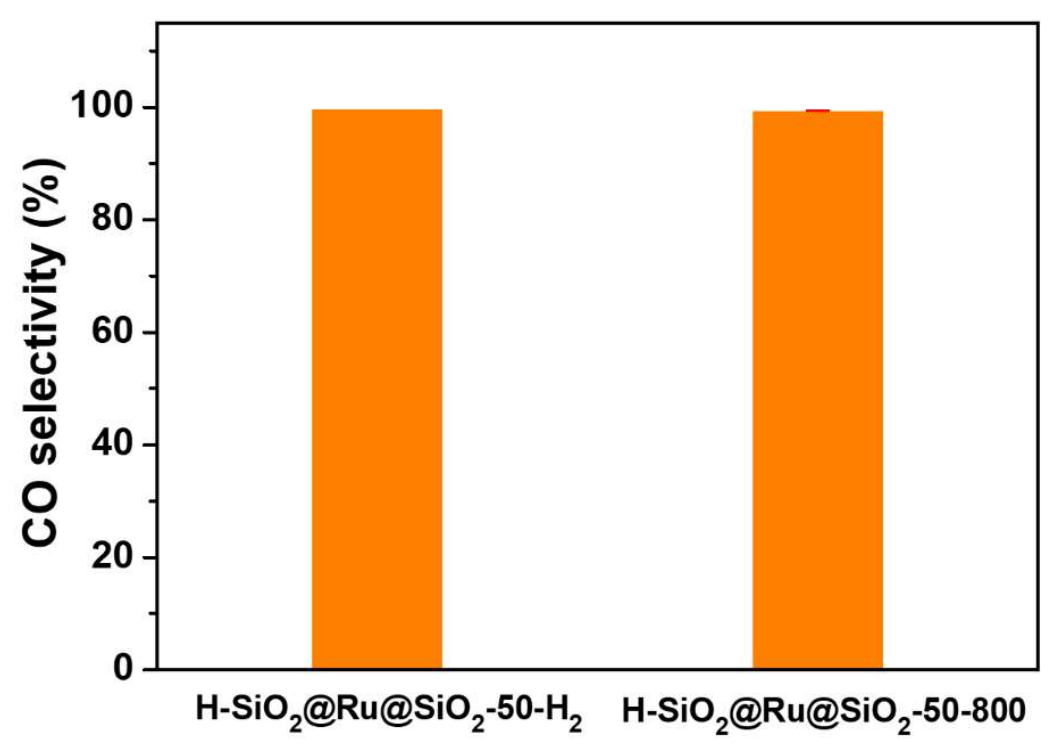

Figure S39. Catalytic selectivity comparison between $\mathrm{H}-\mathrm{SiO}_{2} @ \mathrm{Ru} @ \mathrm{SiO}_{2}-50-\mathrm{H}_{2}$ and $\mathrm{H}-\mathrm{SiO}_{2} @ \mathrm{Ru} @ \mathrm{SiO}_{2}-50-800$. 
Table S1. Comparison of Ru-based catalysts for hydrogenation of $\mathrm{CO}_{2}$.

\begin{tabular}{cccccc}
\hline catalyst & $\begin{array}{c}\text { Loading }^{\mathrm{a}} \\
(\%)\end{array}$ & $\begin{array}{c}\mathrm{Temp} \\
\left({ }^{\circ} \mathrm{C}\right)\end{array}$ & $\begin{array}{c}\mathrm{CO} \text { sel. } \\
(\%)\end{array}$ & TOF $\left(\mathrm{s}^{-1}\right)$ & Ref. \\
\hline $0.4 \% \mathrm{Ru} @ \mathrm{mSiO}_{2}-\mathrm{N}$ & 0.4 & 400 & 93.4 & $0.127^{\mathrm{c}}$ & 1 \\
$1.6 \% \mathrm{Ru} @ \mathrm{mSiO}_{2}-\mathrm{N}$ & 1.6 & 400 & 83.5 & $0.053^{\mathrm{c}}$ & 1 \\
$2.7 \% \mathrm{Ru} @ \mathrm{mSiO}_{2}-\mathrm{N}$ & 2.7 & 400 & 72.4 & $0.035^{\mathrm{c}}$ & 1 \\
$1.4 \% \mathrm{Ru} / \mathrm{TiO}_{2}$ & 1.4 & 200 & 0 & $0.025^{\mathrm{c}}$ & 2 \\
$2.0 \% \mathrm{Ru} / \mathrm{SiO}_{2}$ & 2 & 350 & 47 & $0.054^{\mathrm{c}}$ & 3 \\
$0.1 \% \mathrm{Ru} / \mathrm{Al}_{2} \mathrm{O}_{3}$ & 0.1 & 350 & 34.8 & $0.112^{\mathrm{c}}$ & 4 \\
$\left(\mathrm{Co} 0.95 \mathrm{Ru}_{0.05}\right)_{3} \mathrm{O}_{4}$ & 5.0 & 300 & 1 & - & 5 \\
$\mathrm{Ce} \mathrm{e}_{0.99} \mathrm{Ru} 0.01 \mathrm{O}_{2}$ & 1.0 & 500 & 10 & $0.12^{\mathrm{c}}$ & 6 \\
$\mathrm{Ru} / \mathrm{FeO}_{\mathrm{x}}$ & 0.01 & 250 & 100 & $0.043^{\mathrm{b}}$ & 7 \\
$\mathrm{Ru} / \mathrm{a}-\mathrm{TiO}_{2}$ & 1 & 300 & 99 & - & 8 \\
$\mathrm{Ru} / \mathrm{MnO}_{\mathrm{x}}$ & 5 & 300 & 10 & $0.5^{\mathrm{b}}$ & 9 \\
$\mathrm{Ru} / \mathrm{CeO}_{2}$ & 0.5 & 260 & 90 & $0.0055^{\mathrm{b}}$ & 10 \\
$\mathrm{H}-\mathrm{SiO}_{2} @ \mathrm{Ru} @ \mathrm{SiO}_{2}-30$ & 0.48 & 350 & 99.4 & $0.109^{\mathrm{b}}$ & This work \\
$\mathrm{H}-\mathrm{SiO}_{2} @ \mathrm{Ru} @ \mathrm{SiO}_{2}-30$ & 0.48 & 400 & 99.3 & $0.262^{\mathrm{b}}$ & This work \\
\hline
\end{tabular}

${ }^{a} \mathrm{Ru}$ loading was measured by ICP-MS.

${ }^{\mathrm{b}}$ TOF was calculated as number of $\mathrm{CO}_{2}$ converted per $\mathrm{Ru}$ active site per second.

${ }^{\mathrm{c}}$ TOF was calculated as mole of $\mathrm{CO}_{2}$ converted per mole of total metal per second.

\section{Reference}

(1) Dou, J.; Sheng, Y.; Choong, C.; Chen, L.;Zeng, H. C. Silica nanowires encapsulated Ru nanoparticles as stable nanocatalysts for selective hydrogenation of $\mathrm{CO}_{2}$ to CO. Appl. Catal. B 2017, 219, 580-591.

(2) Li, C.; Zhang, S.; Zhang, B.; Su, D.; He, S.; Zhao, Y.; Liu, J.; Wang, F.; Wei, M.; Evans, D. G., et al. Photohole-oxidation-assisted anchoring of ultra-small $\mathrm{Ru}$ clusters onto $\mathrm{TiO}_{2}$ with excellent catalytic activity and stability. J. Mater. Chem. A 2013, 1, 2461-2467.

(3) Scire, S.; Crisafulli, C.; Maggiore, R.; Minico, S.;Galvagno, S. Influence of the support on $\mathrm{CO}_{2}$ methanation over Ru catalysts: an FT-IR study. Catal. Lett. 1998, 51, 41-45.

(4) Kwak, J. H.; Kovarik, L.;Szanyi, J. $\mathrm{CO}_{2}$ Reduction on Supported $\mathrm{Ru} / \mathrm{Al}_{2} \mathrm{O}_{3}$ Catalysts: Cluster Size Dependence of Product Selectivity. ACS Catal. 2013, 3, 2449-2455.

(5) Zhu, Y.; Zhang, S.; Ye, Y.; Zhang, X.; Wang, L.; Zhu, W.; Cheng, F.;Tao, F. Catalytic Conversion of Carbon Dioxide to Methane on Ruthenium-Cobalt Bimetallic Nanocatalysts and Correlation between Surface Chemistry of Catalysts under Reaction Conditions and Catalytic Performances. ACS Catal. 2012, 2, 2403-2408.

(6) Sharma, S.; Hu, Z.; Zhang, P.; McFarland, E. W.;Metiu, H. $\mathrm{CO}_{2}$ methanation on Ru-doped ceria. J. Catal. 2011, 278, 297-309.

(7) Zhang, D.; Luo, J.; Wang, J.; Xiao, X.; Liu, Y.; Qi, W.; Su, D. S.;Chu, W. Ru/FeOx catalyst performance design: Highly dispersed Ru species for selective carbon dioxide hydrogenation. Chin. J. Catal. 2018, 39, 157-166.

(8) Li, X.; Lin, J.; Li, L.; Huang, Y.; Pan, X.; Collins, S. E.; Ren, Y.; Su, Y.; Kang, L.; Liu, X., et al. Controlling $\mathrm{CO}_{2}$ Hydrogenation Selectivity by Metal-Supported Electron Transfer. Angew. Chem. Int. Ed. 2020, 59, 19983-19989.

(9) Dreyer, J. A. H.; Li, P.; Zhang, L.; Beh, G. K.; Zhang, R.; Sit, P. H. L.;Teoh, W. Y. Influence 


\section{Supporting Information}

of the oxide support reducibility on the $\mathrm{CO}_{2}$ methanation over Ru-based catalysts. Appl. Catal. B 2017, 219, 715-726.

(10) Aitbekova, A.; Wu, L.; Wrasman, C. J.; Boubnov, A.; Hoffman, A. S.; Goodman, E. D.; Bare, S. R.;Cargnello, M. Low-Temperature Restructuring of $\mathrm{CeO}_{2}$-Supported $\mathrm{Ru}$ Nanoparticles Determines Selectivity in $\mathrm{CO}_{2}$ Catalytic Reduction. J. Am. Chem. Soc. 2018, 140, 13736-13745. 
Table S2. Properties and catalytic performance of different catalysts

\begin{tabular}{|c|c|c|c|c|c|c|}
\hline Catalyst & $\begin{array}{c}\text { Shell } \\
\text { thickness } \\
\text { (nm) }\end{array}$ & $\begin{array}{c}\mathrm{Ru} \\
\text { loading } \\
(\%)\end{array}$ & $\begin{array}{l}\text { Temperature } \\
\left({ }^{\circ} \mathrm{C}\right)\end{array}$ & $\begin{array}{c}\mathrm{CO} \\
\text { selectivity } \\
(\%)\end{array}$ & $\begin{array}{c}\text { Conversion } \\
\text { rate } \\
(\mathrm{mol} / \mathrm{g} / \mathrm{h})\end{array}$ & $\begin{array}{c}\text { Conversion } \\
\text { degree } \\
(\%)\end{array}$ \\
\hline \multirow[t]{5}{*}{$\mathrm{H}-\mathrm{SiO}_{2} @ \mathrm{Ru}$} & 0 & 0.99 & 200 & 83.9 & 0.04 & $3.2 \%$ \\
\hline & & & 250 & 83.6 & 0.26 & $3.3 \%$ \\
\hline & & & 300 & 85.4 & 1.46 & $3.9 \%$ \\
\hline & & & 350 & 83.8 & 4.50 & $6.0 \%$ \\
\hline & & & 400 & 83.1 & 10.15 & $9.5 \%$ \\
\hline \multirow[t]{5}{*}{$\mathrm{H}-\mathrm{SiO}_{2} @ \mathrm{Ru} @ \mathrm{SiO}_{2}-30$} & 30 & 0.48 & 200 & 100 & 0.05 & $<1 \%$ \\
\hline & & & 250 & 99.5 & 0.19 & $<1 \%$ \\
\hline & & & 300 & 99.4 & 0.86 & $1.2 \%$ \\
\hline & & & 350 & 99.4 & 3.08 & $1.9 \%$ \\
\hline & & & 400 & 99.3 & 7.40 & $3.7 \%$ \\
\hline \multirow[t]{5}{*}{$\mathrm{H}-\mathrm{SiO}_{2} @ \mathrm{Ru} @ \mathrm{SiO}_{2}-50$} & 50 & 0.27 & 200 & 100 & 0.05 & $2.2 \%$ \\
\hline & & & 250 & 100 & 0.32 & $2.7 \%$ \\
\hline & & & 300 & 99.7 & 1.72 & $3.2 \%$ \\
\hline & & & 350 & 99.8 & 5.28 & $3.9 \%$ \\
\hline & & & 400 & 99.7 & 9.72 & $4.6 \%$ \\
\hline $\begin{array}{c}\mathrm{H}-\mathrm{SiO}_{2} @ \mathrm{Ru} @ 800 \\
8 \mathrm{SiO}_{2}-50-\end{array}$ & 50 & 0.27 & 400 & 99.4 & 4.26 & $2.2 \%$ \\
\hline
\end{tabular}


Table S3. Ru dispersity of different catalysts

\begin{tabular}{|c|c|c|c|}
\hline Catalyst & $\mathrm{H}_{2}$ uptake $^{\mathrm{a}}$ & $\mathrm{Ru}$ dispersion ${ }^{\mathrm{b}}$ & Size Range of $\mathrm{Ru}^{\mathrm{c}}$ \\
\hline $\mathrm{H}-\mathrm{SiO}_{2} @ \mathrm{Ru}-\mathrm{H}_{2}$ & 28.3 & $58 \%$ & $1.0 \sim 6.0 \mathrm{~nm}$ \\
\hline spent $\mathrm{H}-\mathrm{SiO}_{2} @ \mathrm{Ru}-\mathrm{H}_{2}$ & 9.4 & $17 \%$ & $1.0 \sim 12.0 \mathrm{~nm}$ \\
\hline $\mathrm{H}-\mathrm{SiO}_{2} @ \mathrm{Ru} @ \mathrm{SiO}_{2}-30-\mathrm{H}_{2}$ & 18.7 & $79 \%$ & $0.7 \sim 1.8 \mathrm{~nm}$ \\
\hline spent H-SiO ${ }_{2} @ \mathrm{Ru} @ \mathrm{SiO}_{2}-30-\mathrm{H}_{2}$ & 17.7 & $75 \%$ & $0.6 \sim 1.7 \mathrm{~nm}$ \\
\hline $\mathrm{H}-\mathrm{SiO}_{2} @ \mathrm{Ru} @ \mathrm{SiO}_{2}-50-\mathrm{H}_{2}$ & 11.4 & $85 \%$ & $0.6 \sim 1.7 \mathrm{~nm}$ \\
\hline spent $\mathrm{H}-\mathrm{SiO}_{2} @ \mathrm{Ru} @ \mathrm{SiO}_{2}-50-\mathrm{H}_{2}$ & 10.3 & $77 \%$ & $0.6 \sim 1.8 \mathrm{~nm}$ \\
\hline
\end{tabular}

${ }^{\mathrm{a} U n i t}$ : $\mu$ moles $\mathrm{H}_{2} / \mathrm{g}$ catalyst.

${ }^{\mathrm{b}}$ Calculated based on the adsorption of one $\mathrm{H}$ atom per exposed $\mathrm{Ru}$ atom.

'Particle sizes obtained from TEM images. 